\title{
Understanding dwarf galaxies as galactic building blocks
}

\author{
Monica Tosi \\ INAF - Osservatorio Astronomico di Bologna
}

\begin{abstract}
This is a summary of a general discussion held during the third EuroConference on galaxy evolution. Various observational features of the stellar populations in present-day dwarf galaxies were presented to introduce the discussion on the possibility that these systems be the main building blocks of spiral and elliptical galaxies. Many people in the audience turned out to think that the inconsistencies among the observed properties of large and dwarf galaxies are too many to believe that the former are built up only by means of successive accretions of the latter. However, theorists of hierarchical galaxy formation suggested that presentday dwarfs are not representative of the galactic building blocks, which may be completely invisible nowadays. Some of them suggested that, contrary to what is usually assumed in hierarchical modelling, the actual building blocks were still fully gaseous systems when their major mergers occurred. If this is the case, then most of the inconsistencies can be overcome, and the scenario of hierarchical galaxy formation becomes not too different from that of a slow gas accretion.
\end{abstract}

Keywords: Dwarf galaxies, galaxy evolution, galaxy formation

\section{Introduction}

Observations show that galaxies merge here and there in the local Universe and that big galaxies accrete their satellites. We all know the cases of the Magellanic Stream and Sagittarius which are being accreted by the Milky Way. Andromeda is quite similar in this respect, as shown by Ferguson's et al (2002). Their data on the red giant stars observed in M31 with the INT-WF camera indicate that that system contains streams and clumps just as our own Galaxy.

We learned that Cold Dark Matter (CDM) cosmology predicts that only dark matter halos with mass smaller that $10^{8} M_{\odot}$ can form from $3 \sigma$ fluctuations on primordial density perturbations, and that more massive systems can only form by subsequent merging of these protogalactic fragments. Satellites have therefore a major role and are predicted to continuously interfere with galaxy evolution.

What we want to discuss here is whether dwarfs can be considered as the only building blocks of massive galaxies, or their accretion is a frequent but not necessary event. We should then discuss whether or not dwarfs properties are consistent with a purely hierarchical scenario for galaxy formation. Another important question is what can be the high$\mathrm{z}$ counterparts of local dwarfs, but this is not the topic of this discussion.

(c) 2018 Kluwer Academic Publishers. Printed in the Netherlands. 


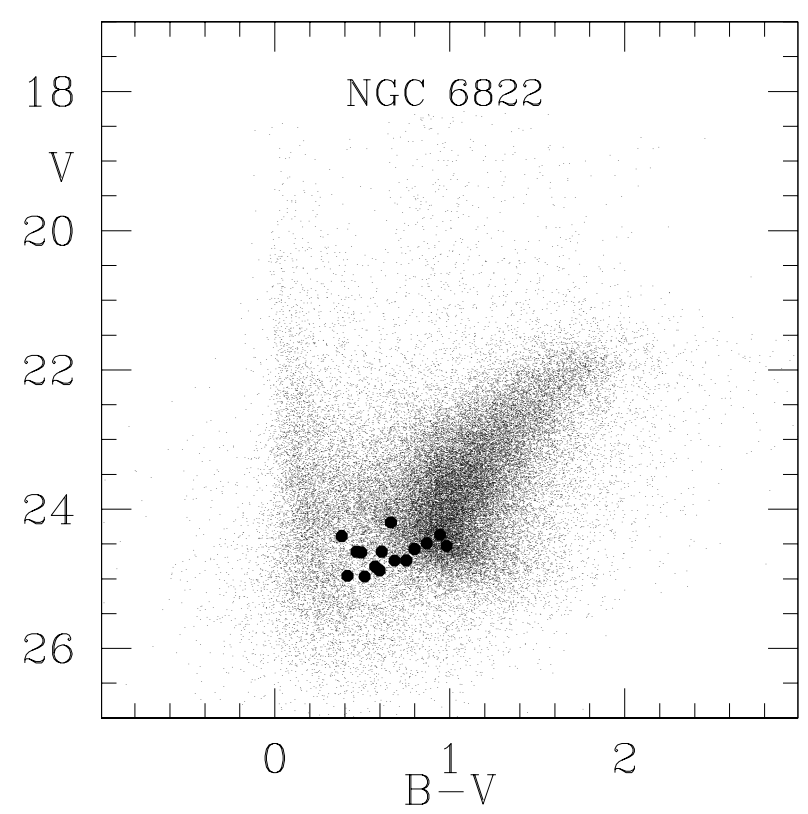

Figure 1. CMD of the dwarf irregular galaxy NGC 6822 as derived from VLT photometry. The heavier points correspond to confirmed RR Lyrae variables (courtesy L. Baldacci).

In the following, I shall first present a list of pro and contra arguments as discussion bases and then briefly summarize the highlights of the actual discussion.

\section{Discussion Arguments}

\subsection{AGES}

For what concerns stellar ages, we expect the first galaxies able to form to contain the oldest stars (see e.g. Steinmetz 2001). Early-type dwarfs clearly contain old stars (e.g. Horizontal Branch, HB, stars, corresponding to ages of 12-15 Gyr), but what about late-type dwarfs ? Well: all the examined ones have revealed the presence of stars as old as reachable by the available photometry. For instance, in the nearby dwarf irregular NGC 6822 the discovery of several RR Lyraes has allowed Baldacci et al (2002) to firmly conclude that HB stars, i.e. 12-15 Gyr old stars, are present (Fig.1)

In more distant galaxies, the reachable stars are obviously intrinsically brighter, and therefore younger. At 10-12 Mpc, even with the HST-WFPC2, in IZw18 we (Aloisi et al 1999) probably reached only 


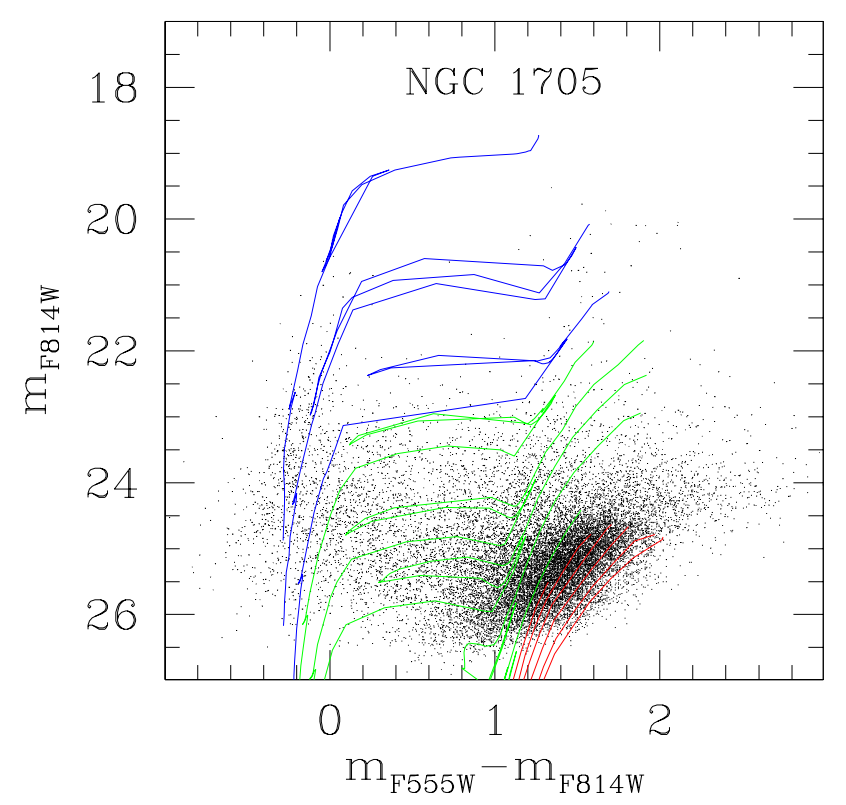

Figure 2. CMD of the BCD galaxy NGC 1705 as derived from HST photometry. Overimposed are the Padova stellar evolutionary tracks from 0.6 to $30 M_{\odot}$ (see Tosi et al 2001 for details).

AGB stars, i.e. less than 1 Gyr old (but the ACS should reach the tip of the red giant branch, RGB). At $5 \mathrm{Mpc}$, with the HST-WFPC2, we (Tosi et al 2001, Annibali et al. 2002) could reach well below the RGB-tip (Fig.2), thus demonstrating that the blue compact dwarf (BCD) NGC 1705 , in addition to very young stars, contains also objects at least 5 Gyr old. Table 1, an update of one kindly provided by U.Hopp, lists all the BCDs whose colour-magnitude diagrams (CMDs) have been derived from HST photometry and have been interpreted in terms of stellar ages and star formation (SF) histories. In all of them, stars as old as allowed by the photometric magnitude limit have been found. Is this sufficient to conclude that all dwarfs contain stars formed in the earliest Universe? The sample, in my opinion, is still too small to reach such a strong conclusion.

In any case, the mean ages of stars in ellipticals are generally older than those in dwarfs, a feature recently confirmed by the study (Kauffmann et al 2002) of 80000 galaxies observed by the Sloan survey. An argument against pure merging galaxy formation, since building blocks should instead contain older stars than their daughters.

An argument which is often presented in favour of hierarchical galaxy formation is the age spread e.g. of the Milky Way halo stars: no doubt 
Table I. BCDs with SFH derived from HST CMDs

\begin{tabular}{l|c|c|l}
\hline Galaxy & D (Mpc) & $12+\log (\mathrm{O} / \mathrm{H})$ & Reference \\
\hline I Zw 18 & $10-12$ & 7.18 & Aloisi et al. 1999 \\
VII Zw 403 & 4.4 & 7.69 & Lynds et al. 1998 \\
UGCA 290 & 6.7 & $?$ & Crone et al. 2002 \\
I Zw 36 & 5.8 & 7.77 & Schulte-Ladbeck et al. 2001 \\
NGC 6789 & 3.6 & $7.7 ?$ & Drozdovsky et al., 2001 \\
UGC 5272 & 5.5 & 7.83 & Hopp et al., in prep \\
MrK 178 & 4.2 & 7.95 & Schulte-Ladbeck et al. 2000 \\
NGC 4214 & 2.7 & 8.27 & Drozdovsky et al. 2002 \\
NGC 1569 & 2.2 & 8.31 & Greggio et al. 1998 \\
NGC 1705 & 5.1 & 8.36 & Annibali et al. 2002 \\
& & & \\
\hline
\end{tabular}

that if halo stars have formed during its collapse and have ages differing by 1-2 Gyr, we cannot let the halo have collapsed in $100 \mathrm{Myr}$, as in the original version of the monolithic collapse scenario (Eggen, LyndenBell, Sandage 1962). But wouldn't a slower collapse be compatible with the observed stellar ages ?

\subsection{Chemical Abundances}

We know that dwarfs (both early- and late-type dwarfs) are metal poor $\left(0.0001 \leq Z \leq Z_{\odot} / 2\right)$. How about abundance ratios which are the signature of the SF timescales ? For instance, the $[\alpha / F e]$ ratio depends on the relative contributions of SNeII ( $\alpha$ producers) and SNeI $(F e$ major producers) so that, at a given metallicity, slow, continuous SFs imply low $[\alpha / F e]$, while short SFs imply high $[\alpha / F e]$ (see Matteucci 1992 and her figure 1 in this volume). In the few local dwarfs where $[\alpha / F e]$ has been measured with appropriate spectroscopy, it ranges between the low ratio -0.2 of the Magellanic Clouds $(\Rightarrow$ continuous $\mathrm{SF}$ ) and the moderate ratio $0.02 \leq[\alpha / F e] \leq 0.13$ of dSph's (Shetrone et al 2001 and Fig.3, Tolstoy, this volume). In the Milky Way halo, the $\alpha$ overabundance is about $0.4-0.5$; in ellipticals it is observed to range between $\sim 0.05$ and 0.25 ( $\Rightarrow$ short SF episodes). How could ellipticals (high $Z$ ) and halos (low $Z$ ) manage to get $[\alpha / F e]>0.2$ if dwarfs similar to the local ones are their building blocks ? In fact, semi-analytical models do not reproduce the whole range of ratios observed in ellipticals (e.g. Thomas 2001) because they fail to get a large portion of its high $[\alpha / F e]$ end. Chemical evolution models based on monolithic collapse 


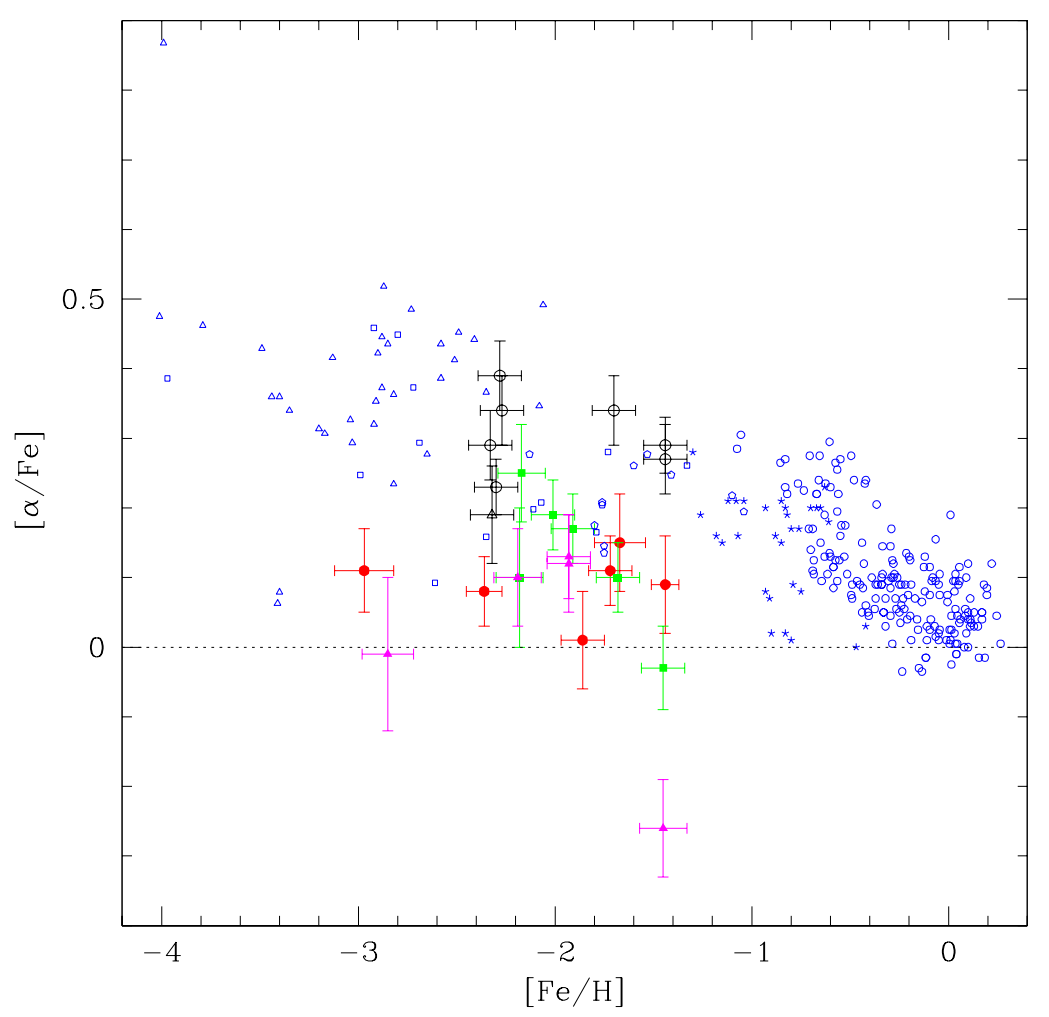

Figure 3. Abundance ratios derived from high-resolution spectra of Ursa Minor, Draco and Sextans (filled dots with error bars) compared with those of Milky Way halo and disk stars (dots) and globular clusters (open symbols with error bars). See Shetrone et al 2001 for details.

do, instead, reproduce the observed ratios (see figure 2 in Matteucci, this volume). If the $\mathrm{Sf}$ is more time-concentrated in ellipticals than in dwarfs, how can the latter be the building blocks of the former?

Metallicity provides another problem for pure hierarchical galaxy formation: the metallicity distribution of halo globular clusters, both in the Galaxy and in ellipticals. Actually, the multimodality of the metallicity distribution functions of globular clusters (e.g. Harris 2001, and references therein) is one of the first arguments against monolithic collapse and in favour of hierarchical formation, buth why are the distributions most frequently bimodal, rather than multimodal, if the accretion episodes are several tens or hundreds and at all possible epochs? 


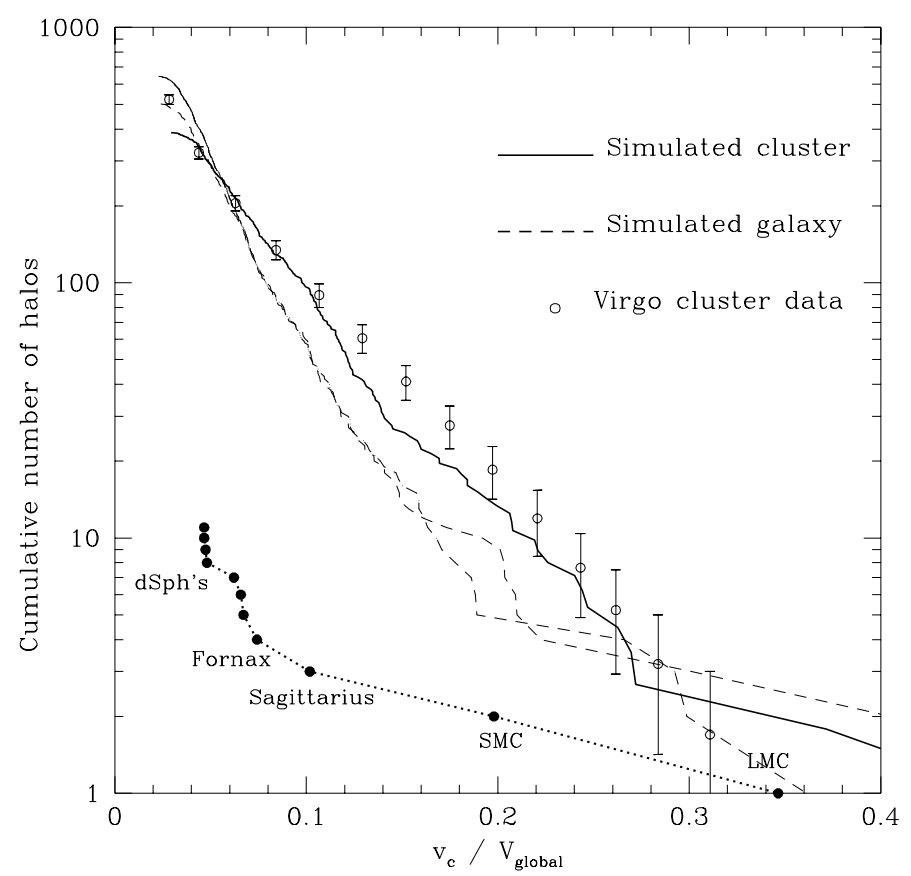

Figure 4. Satellite number as a function of circular velocity for the Galaxy and the Virgo cluster as observed (dots) and as predicted by hierarchical models (dashed and solid lines, for the Galaxy and Virgo respectively). From Moore et al. (1999).

\subsection{Counts}

The number of dwarf satellites predicted by CDM scenarios is much larger than observed: 11 satellites observed against 50-500 (depending on the assumptions) predicted for the Milky Way, and 40 observed against 1000 expected DM halos in the Local Group (see e.g. Mateo 1998, Moore et al 1999). This is the so-called satellite catastrophe for hierarchical galaxy models: where have the missing satellites gone ?

Could they be the most compact high velocity clouds (CHVCs) observed in large number around the Galaxy, as suggested by Blitz et al (1999) ? Many observational campaigns (e.g. Gibson 2001, Simon and Blitz 2002, Hopp, this volume) have been performed to search for stars in these systems, under the hypothesis that if they are the equivalent of the Galactic building blocks they must contain stars. However, no star has ever been found in a HVC, despite the fact that all these campaigns had sufficient sensitivity to detect them.

Could most of the satellites be invisible because their SF has been inhibited by early reionization, as suggested e.g. by MacLow and Fer- 
rara (1999) and Bullock et al. (2000) ? But then, why haven't visible dwarfs been inhibited too?

Or, is the satellite overprediction the consequence of having assumed the dark matter to be cold, while it should actually be assumed to be warm?

\subsection{Miscellanea}

There are several other aspects to discuss to better understand whether or not present-day dwarf galaxies can be considered the analogues of galactic building blocks.

Carbon stars: Present-day dwarfs contain many C-stars, while the halos of big galaxies like the Milky Way don't. If the latter have formed from dwarfs merging, where have their C-stars gone ? Probably, the only way to avoid the overprediction of halo C-stars is to assume that the halo itself formed more than $10 \mathrm{Gyr}$ ago, i.e. before the birth of C-star progenitors (e.g. van den Bergh 1996).

Half-light radii of globular clusters: The half-light radii of globular clusters in the Galactic halo are tightly correlated with their Galactocentric distance, a circumstance suggesting that they are all ruled by a common law. Since the Galactic half-light radii are all larger than those of globular clusters e.g. in the nearby dwarf galaxy Fornax, how can our halo clusters come from the accretion of Fornax-like systems (e.g. van den Bergh 1996) ?

Kinematics: Standard CDM models of hierarchical galaxy formation have several problems in predicting the kinematic properties of present day galaxies. One is the disk overheating due to the energy transferred by the accreted fragments (e.g. Toth and Ostriker 1992, Moore et al. 1999). For instance, Torres et al (2001) suggest that the kinematic properties of white dwarfs in the Galactic disk are compatible only with small accretion episodes (i.e. with satellite mass $\leq 4 \%$ of the Milky Way mass) occurred earlier than $\sim 6$ Gyr ago, and are definitely inconsistent with more recent (in the last 6 Gyr) or massive (satellite with mass $\geq 16 \%$ of Milky Way mass) ones.

The so-called angular momentum catastrophe is probably the worst kinematic problem: the dynamical friction of the orbiting gas clumps and the gravitational torques exerted by non-spherical DM distributions make the angular momenta predicted by hierarchical scenarios for spiral disks more than 10 times lower than observed and no convincing way out of this inconsistency has been found yet (e.g. Navarro and Steinmetz, 2000). 
Monica Tosi

\section{Discussion}

The topics described above were extensively debated during a very lively discussion, with an audience fairly well balanced between supporters of the hierarchical and of the monolithic schools of thought. We defined as hierarchical all the models (CDM, WDM, etc.) predicting that galaxies form by successive merging of lower mass fragments, which inevitably imply that more massive galaxies form later than lower mass ones, and as monolithic all the models assuming galaxies of any size to form from the collapse of one or more gas clouds, which does not imply any delay in the formation of massive systems.

We all agreed that some of the age, chemical and dynamical inconsistencies between the observed properties of present-day dwarf galaxies and those of big galaxies make dwarfs very unlikely to be the major building blocks of systems like the Milky Way and normal ellipticals. It was however argued (by M. Steinmetz) that this is not an argument against hierarchical galaxy formation theories, but evidence that present-day dwarfs are simply not the local counterpart of the building blocks of the theoretical CDM models. In his opinion the actual building blocks may be completely invisible nowadays and different from any kind of known systems. This may sound as a fairly ad hoc way out of the problem, but we cannot exclude it. It requires, at any rate, that these unknown systems either don't form stars at all before merging or form them with the appropriate characteristics.

One of the most interesting novelties of this debate was the assertion (e.g. by S. Lamb) that in some hierarchical galaxy formation models the creation of large galaxies by major mergers of small fragments occurs when the baryonic matter of the latter is still fully gaseous. In this case, most of the problems of published CDM galaxy formation models would be overcome: the stars of any galaxy would form in situ just as in the monolithic scenario. The whole question would then concern the timescales for the formation of galaxies of different size and morphological type. If some of the cold and warm dark matter models already allow for early formation even of big ellipticals, as stressed by some of the participants (e.g. R. Dominguez), we may dare foreseeing that, hopefully soon, the two schools of thought will converge in a compromising scenario, where both the cosmological and the local properties derived from observations will be reproduced by theoretical models.

tosikiel.tex; 2/11/2018; $18: 00 ;$ p.8 


\section{Acknowledgements}

I thank Ulrich Hopp for providing the data of Table 1 and for very interesting conversations on BCDs. Lara Baldacci and Gisella Clementini kindly provided their results on NGC 6822 in advance of publication, and Matthew Shetrone made his figure available in convenient format. This work has been partially supported by the Italian ASI and MIUR through contracts ASI-I/R/35/00 and Cofin-2000.

\section{References}

Aloisi, A., Tosi, M., Greggio, L. The Star Formation History of I ZW 18 AJ, 118: 302-322, 1999

Annibali, F., Tosi, M., Greggio, L., Aloisi, A., and Leitherer, C. The star formation history of NGC1705. AJ submitted, 2002

Baldacci, L., Rizzi, L., Clementini, G., Held, E.V., Momany, Y. The distance to NGC6822 from its RR Lyrae's. in New Horizons in globular cluster astronomy, G.Piotto, G.Meylan, G.Djorgowski, M.Riello eds, ASP Conf.Ser., 2002 in press

Blitz, L., Spergel, D.N., Teuben, P.J., Hartmann, D., Burton, W.B. High-Velocity Clouds: Building Blocks of the Local Group. ApJ, 514: 818-843, 1999

Bullock, J.S., Kravtsov, A.V., Weinberg, D.H. Reionization and the Abundance of Galactic Satellites. ApJ, 539: 517-521, 2000

Crone, M.M., Schulte-Ladbeck, R.E., Greggio, L., Hopp, U. The Star Formation History of the Blue Compact Dwarf Galaxy UGCA 290. ApJ, 567: 258-276, 2002

Drozdovsky, I.O., Schulte-Ladbeck, R.E., Hopp, U., Crone, M.M., Greggio, L. The Stellar Content of NGC 6789, A Blue Compact Dwarf Galaxy in the Local Void. ApJ, 551: L135-L138, 2001

Drozdovsky, I.O., Schulte-Ladbeck, R.E., Hopp, U., Greggio, L., Crone, M.M. The Dwarf Irregular/Wolf-Rayet Galaxy NGC 4214. AJ, 124: 811-827, 2002

Eggen, O. J.; Lynden-Bell, D.; Sandage, A. R. Evidence from the motions of old stars that the Galaxy collapsed. ApJ, 136: 748-766, 1962

Ferguson, A.M.N., Irwin, M.J., Ibata, R.A., Lewis, G.F., Tanvir, N.R. Evidence for Stellar Substructure in the Halo and Outer Disk of M31. AJ, 124: 1452-1463 ,2002

Gibson, B.K. The Infall of Gas onto the Galactic Disk. The dynamics, structure and History of Galaxies, G.S.Da Costa and E.M.Sadler eds, ASP Conf.Ser, astro$\mathrm{ph} / 0112206,2002$ in press

Greggio, L., Tosi, M., Clampin, M., De Marchi, G., Leitherer, C., Nota, A., Sirianni, M. The Resolved Stellar Population of the Poststarburst Galaxy NGC 1569. ApJ, 504: 725-742, 1998

Harris, W.E. Globular cluster systems. Saas-Fee Advanced Course 28, L.Labhardt and B.Binggeli eds (Springer-Verlag), p.223-, 2001

Kaufmann, G. et al. The dependence of star formation history and internal structure on stellar mass for 80,000 low-redshift galaxies. MNRAS submitted, astro-ph/0205070, 20022002 
Lynds, R., Tolstoy, E., O'Neil., E.J., Jr., Hunter, D.A. Star Formation in and Evolution of the Blue Compact Dwarf Galaxy UGC 6456 Determined from HST Images. AJ, 116: 146-162, 1998

Mac Low, M.-M., and Ferrara, A. Starburst-driven Mass Loss from Dwarf Galaxies: Efficiency and Metal Ejection. ApJ, 513: 142-155, 1999

Mateo, M.L. Dwarf Galaxies of the Local Group. ARAA, 36: 435-506, 1998

Matteucci, F. The evolution of the alpha-elements in galaxies. MemSAIt, 63, 301314, 1992

Moore, B., Ghigna, S., Governato, F., Lake, G., Quinn, T., Stadel, J., Tozzi, P. Dark Matter Substructure within Galactic Halos. ApJ, 524: L19-L22, 1999

Navarro, J.F., and Steinmetz, M. Dark Halo and Disk Galaxy Scaling Laws in Hierarchical Universes. ApJ, 538: 477-488, 2000

Schulte-Ladbeck, R.E., Hopp, U., Greggio, L., Crone, M.M. A Near-Infrared Stellar Census of Blue Compact Dwarf Galaxies: NICMOS Detection of Red Giant Stars in the Wolf-Rayet Galaxy Markarian 178. AJ, 120: 1713-1730, 2000

Schulte-Ladbeck, R.E., Hopp, U., Greggio, L., Crone, M.M., Drozdovsky, I.O. A Near-Infrared Stellar Census of Blue Compact Dwarf Galaxies: The Wolf-Rayet Galaxy I Zw 36. AJ, 121: 3007-3025, 2001

Shetrone, M.D., Ct, P., and Sargent, W.L.W. Abundance Patterns in the Draco, Sextans, and Ursa Minor Dwarf Spheroidal Galaxies. ApJ, 548: 592-608, 2001

Simon, J.D. and Blitz, L. The Absence of Stars in Compact High-Velocity Clouds. ApJ, 574: 726-739, 2002

Steinmetz, M. The Hierarchical Origin of Galaxy Morphologies. in Dwarf galaxies and their environment, K.S. De Boer, R. Dettmar, U. Klein eds (Shaker Verlag), p.297, 2001

Thomas, D. Abundance ratios in hierarchical galaxy formation. in The evolution of galaxies. I-Observational clues, J.M. Vilchez, G.Stasinska, E.Perez eds (Kluwer), ApSSS, 277: 209-209, 2001

Torres, S., Garca-Berro, E., Burkert, A., Isern, J. The impact of a merger episode in the galactic disc white dwarf population. MNRAS, 328: 492-500, 2001

Tosi, M., Sabbi, E., Bellazzini, M., Aloisi, A., Greggio, L., Leitherer, C., Montegriffo, P. The resolved stellar populations in NGC 1705. AJ, 122: 1271-1288, 2001.

Toth, G., and Ostriker, J.P. Galactic disks, infall, and the global value of Omega. ApJ, 389: 5-26, 1992

van den Bergh, S. Formation of the Galaxy. PASP, 108: 986-995, 1996 